\title{
¿Estamos los pediatras preparados para detectar y prevenir los problemas psicológicos de nuestros pacientes?
}

\author{
M. Duelo Marcos ${ }^{a}$, ML.Arroba Basanta ${ }^{b}$ \\ aPediatra. CS Guayaba. Madrid. España. \\ 'Pediatra. CS Pozuelo Estación. Pozuelo de Alarcón, Madrid. España.
}

\begin{abstract}
Si introducimos "salud mental infantil" en un buscador de Internet encontramos más de cuatro millones de "entradas" relacionadas en castellano. La prensa escrita y la televisión (Supernanny, Hermano mayor, etc.) también reflejan el interés creciente y la preocupación social por
\end{abstract} este tema.

La prevalencia de trastornos mentales de niños y adolescentes varía entre el 5 y el $22 \%$, dependiendo de la metodología, tipo de entrevista, muestras e inclusión del criterio de discapacidad. Entre un 4 y $6 \%$ de los niños y adolescentes padece un trastorno mental grave ${ }^{1}$.

Cuando hablamos de manera informal con colegas sobre las dificultades del trabajo diario en Atención Primaria (AP) pediátrica, más pronto que tarde sale el asunto de lo difícil que es abordar la morbilidad psicosocial. Asistimos a un aumento de conflictos en todas las áreas de la vida infantil y adolescente: alimentación (obesidad, anorexia, bulimia...), violencia (entre escolares, contra los padres o los profesores...), alcohol, tabaco y otras drogas (inicio del consumo cada vez más precoz, aumento de los comas etílicos y de intoxicaciones por drogas de diseño sobre todo en fin de semana...), familia (divorcios muy mal resueltos con la correspondiente repercusión en los hijos, maltrato a la mujer y a los niños...), educación (fracaso escolar, poca tolerancia y búsqueda del placer inmediato, búsqueda de modelos en lo audiovisualbasura...), sexualidad (aumento de los abusos sexuales, de los embarazos no

Mar Duelo Marcos, mmduelo@pap.es

Los autores declaran no presentar conflictos de intereses en relación con la preparación y publicación de este artículo. 
deseados y de sus interrupciones, de padres adolescentes, precocidad en las primeras relaciones genitales...), social (comportamientos inaceptables normalizados, como botellones, desprecio a las normas de tolerancia y cortesía...).

Es al equipo de Pediatría de AP (enfermero/a y pediatra), a los que acuden los padres preocupados, o empujados por el profesor cuando se dan cuenta del conflicto. Cada vez nos enfrentamos a más consultas de la esfera emocional (rabietas, celos, problemas de relación con sus pares, tiranos de sus padres, etc.), y a síntomas para los que no encontramos razón orgánica: de alimentación, sueño, dolores abdominales, cefaleas, taquicardias, etc., ya que el problema está en la esfera de lo psicológico.

Lo nuestro, lo de los pediatras de AP, es la prevención y la detección precoz, y en este campo podemos hacer mucho. La interacción entre la genética y el ambiente va a condicionar el desarrollo biopsicosocial del niño, y para que se desarrolle adecuadamente una parte esencial ocurre en los primeros años de la vida y es el vínculo entre el niño y la persona que le cuida, generalmente la madre, lo que le hace crecer física y mentalmente. El tipo de vínculo, aunque no es el único, es uno de los aspectos primordiales condicionantes tanto para el desa- rrollo psicológico adecuado del niño, como para la instauración de patologías. Cuando el vínculo falla, aparecen dificultades físicas y psíquicas, dificultades que si no se resuelven, lejos de mejorar con el tiempo se agravan o se añaden nuevas dificultades.

La edad de la maternidad ha cambiado, las pautas educativas también. ¿Qué esta pasando? La crianza parece haberse complicado y los niños no vienen con un "manual de instrucciones" al nacer. Los horarios laborales extensos facilitan que, cuando los padres llegan a casa, no quieran contrariar al niño sino "disfrutar de él o ella". Son incapaces de poner límites y ejercer su autoridad como padres. Y se encuentran con niños llorones, desordenados o mal comedores que no les dejan dormir por la noche ni descansar. Los padres, culpabilizados en ocasiones por su ausencia, se convierten en auténticos "parques de atracciones" para sus hijos.

Es en este momento, durante los primeros años de la vida del niño dónde el equipo de pediatría, desde nuestra posición de acompañamiento a lo largo del tiempo podemos realizar prevención y detección precoz: ya en las primeras consultas podemos diagnosticar problemas graves, como las psicosis puerperales o las depresiones posparto, que serán motivo de derivación urgente a psiquia- 
tría; las grandes dificultades para establecer un buen vínculo y ofrecer cuidados adecuados al niño, que pueden expresarse como trastornos de alimentación, sueño, etc.; o las dificultades familiares, los llamados "casos sociales". Es también durante el primer año de vida cuando se manifiesta la patología grave, como el autismo y la psicosis, y en todos el diagnóstico y tratamiento precoz es fundamental para el futuro mental del niño y de la familia.

Las dificultades en las pautas de crianza son los casos que, sin ser graves, ocupan mucho tiempo en las consultas. Hace años existía cierto rechazo a que los pediatras enviáramos a los niños y sus familias al psicólogo o al psiquiatra. Esa actitud ha pasado a la historia: los padres demandan esa derivación con frecuencia creciente. $\mathrm{Y}$ no solo eso, sino que también por temas educativos (fracaso escolar, dificultades de aprendizaje), "mala" conducta (niños desobedientes, problemas con el aprendizaje de hábitos de alimentación, del sueño, etc.), duelos normales (muertes, etc.), que inundan las consultas de Salud Mental retrasando la asistencia de casos realmente psiquiátricos.

Otro aspecto que llama la atención es que muchas familias han dimitido de su función educadora y nos lo dicen claramente en la consulta: ya les enseñan en la guardería a comer solos, el control de esfínteres, a jugar, a ordenar, etc., y ya les enseñarán en el colegio a estudiar y hacer los deberes... Y es, en estos casos, cuando es primordial tener claro que los trastornos de alimentación, sueño y otros que se derivan a los cuatro, seis, diez años, tienen un punto de partida en el comienzo de la interacción madrebebé.

La prevención de la patología infantil pasa por entender la interconexión de "lo mental" con "lo físico" y "lo social", y cómo ocurre en los primeros años de vida, con las primeras relaciones con y entre los padres; es en ese momento cuando se ponen los cimientos de lo que será la futura salud mental.

Todo el mundo psicológico de la recién madre tiene que reorganizarse. Durante el embarazo, incluso antes, se forma de manera inconsciente un "bebé imaginado", y al dar a luz la madre tiene que dar cabida al "bebé real", al que tiene en sus brazos y a su nueva condición, el paso de "hija" a "madre". Vivimos en una sociedad competitiva y con prisas, las familias desean un niño sano en todos los aspectos. Ese nivel de exigencia no siempre se corresponde con la realidad.

Para una madre el pediatra es una figura con un gran poder, en quien confía para calmar sus angustias ante la respon- 
sabilidad de la crianza del bebé. El pedia- el periodo de formación como especialistra, para establecer un vínculo adecuado tas, se insistió poco en todos estos ascon la madre, debe preguntar por el pro- pectos. Nos hemos "autoformado" ceso del parto y posparto, escuchar du- como hemos podido. Además y como en das o quejas y observar como coge al cualquier otra área de la Pediatría, la bebé, qué contacto tiene con él, dónde morbilidad relacionada con la salud le coloca, si está ansiosa, deprimida, etc. mental ha cambiado a lo largo de los Esta escucha y mirada en las primeras años.

consultas proporcionará una base de Nos gustaría que este número de la confianza y seguridad de la madre hacia Revista Pediatría de Atención Primaria la consulta de Pediatría.

En fin, y por último: los pediatras nos sentimos un poco desbordados. Durante en el que encontramos varios temas de Psiquiatría y Psicología ${ }^{2,3}$ reflejara esta preocupación y nos ayudara a todos.

\section{Bibliografía}

1. Flora de la Barra M. Epidemiología de trastornos psiquiátricos en niños y adolescentes: Estudios de prevalencia. Rev Chil Neuro-Psiquiat. 2009;47(4):303-14.
2. Ortiz Soto $P$, Duelo Marcos $M$, Escribano Ceruelo E. La entrevista en salud mental infantojuvenil. Rev Pediatr Aten Primaria. 2011;13:64560.

3. Armero Pedreira P, Bernardino Cuesta B, Bonet De Luna $C$. Acoso escolar. Rev Pediatr Aten Primaria. 2011;13:661-70. 